$\$$ Research Square

\title{
Evaluation of percutaneous endoscopic lumbar discectomy in treatment of obese adolescents with lumbar disc herniation $₫ A$ retrospective study
}

Haijiang Yu

Peking University Third Hospital

Bin Zhu

Capital Medical University Affiliated Beijing Friendship Hospital

Xiaoguang Liu ( $\sim$ xgliudoctor@163.com )

Peking University Third Hospital

\section{Research Article}

Keywords: adolescent, obesity, lumbar disc herniation, percutaneous endoscopic lumbar discectomy, minimally invasive surgery

Posted Date: April 13th, 2021

DOI: https://doi.org/10.21203/rs.3.rs-389600/v1

License: (1) This work is licensed under a Creative Commons Attribution 4.0 International License. Read Full License 


\section{Abstract}

Background: Obese patients are at risk of complications such as poor wound healing and increased infection rates after spinal surgery. Percutaneous endoscopic lumbar discectomy (PELD) has advantages over conventional open surgery in the treatment of obese adult patients with lumbar disc herniation (LDH) because it can decrease perioperative complications and enhance satisfaction degrees of patients. However, no clinical studies have evaluated the efficacy of PELD in obese adolescents with LDH. This study aimed to evaluate the efficacy of PELD in the treatment of obese ALDH.

Methods: We retrospectively collected clinical data from 208 patients with single-segment ALDH who underwent PELD treatment in our hospital between January 2015 and December 2019. According to the body mass index classification standard of obesity for adolescents in our country, the patients were divided into obese and non-obese groups (control group). Based on the preoperative baseline data of the two groups, propensity score matching was performed to select patients from the two groups for the comparative study. Perioperative data included operative time, intraoperative blood loss and length of postoperative hospitalization. The visual analog scale (VAS), Oswestry disability index (ODI) and modified MacNab criteria were recorded as the main indicators of the surgical outcome, and the recurrence rate and incidence of complications were recorded as the minor indicators.

Results: The obese and control groups included 45 patients each after 1:1 propensity score matching. Both groups showed improvements in VAS and ODI scores after surgery and at each follow-up time point $(p<0.05)$. However, there was no significant statistical difference in VAS and ODI scores between the two groups at each follow-up time point ( $p>0.05)$. In addition, the differences in operative time, intraoperative blood loss, length of postoperative hospitalization, incidence of complications, and recurrence rate were not statistically significant between the two groups $(p>0.05)$. At the final follow-up, there was no significant difference in the excellent and good rate of MacNab classification between the two groups $(p>0.05)$.

Conclusion: PELD is a safe and effective minimally invasive technique for the treatment of obese patients with ALDH. The efficacy of PELD in obese and non-obese patients with ALDH was comparable.

\section{Background}

Lumbar disc herniation (LDH) is the most common cause of lower back and leg pain in adults. However, LDH is rare in children and adolescents. The incidence rate reported in the literature was $0.6-6.8 \%[1,2]$. However, with improvements in living standards and changes in modern living habits, the incidence of LDH is increasing every year and tends to occur at a younger age [3]. Overweight or obesity is an important risk factor for the onset of adolescent lumbar disc herniation (ALDH), and the proportion of obese patients with ALDH is also increasing [4].

The increasing incidence of obesity has become a serious public health problem worldwide. It has been reported that obese adults who undergo spinal surgery have a higher incidence of surgical complications and more surgical blood loss than non-obese patients [5-7]. Compared with traditional open surgery, minimally invasive spinal surgery such as percutaneous endoscopic lumbar discectomy (PELD), has more advantages such as less surgical trauma and faster postoperative recovery. Cole et al. [8] believed that its application in 
obese adults could reduce the length of surgical incision and the occurrence of complications such as infection, thereby improving clinical efficacy and patient satisfaction. However, no studies have evaluated the short- or mid-term efficacy of PELD in obese adolescents with LDH. Therefore, we conducted a retrospective follow-up study of obese adolescents with LDH who underwent PELD at our hospital for 1 to 5 years. Our study focused on the outcomes and safety of PELD in the treatment of obese patients with ALDH.

\section{Methods}

\section{Patients}

We retrospectively analyzed patients with ALDH who underwent PELD in our hospital between January 2015 and December 2019. The diagnosis of LDH was confirmed based on medical history, physical examination, magnetic resonance imaging (MRI) of the lumbar intervertebral disc, and surgical records. The inclusion criteria included patients with the following: (1) age $\leq 21$ years, (2) LDH that was clearly diagnosed by MRI in a single segmental spine, and the clinical manifestations of patients were consistent with imaging findings, (3) no significant improvement of symptoms after 6 weeks of standard conservative treatment, and (4) surgery performed by a senior surgeon (with $>2$ years of experience in spinal surgery). The inclusion criteria included patients with the following: (1) lumbar disc herniation in multi-segmental spine; (2) other previous lumbar spine surgeries; (3) spinal fractures, lumbar spondylolisthesis, spinal tumors, scoliosis, or some other orthopedic specialty diseases such as spinal tuberculosis or infection; or (4) severe medical diseases and mental disorders.

A total of 208 patients with ALDH met the above criteria. According to the body mass index (BMI) classification criteria for obese adolescents under 18 years of age in our country (Fig. 1) and the BMI classification criteria for obese adolescents over 18 years old $\left(B M I \geq 28 \mathrm{~kg} / \mathrm{m}^{2}\right)$, these patients were divided into obese and non-obese groups (control group). To ensure the balance and comparability of the clinical data between the two groups and reduce the confounding bias, propensity score matching (PSM) was performed based on the preoperative baseline data.

\section{Propensity score matching (PSM)}

To reduce the impact of potential confounding factors, the patients in the obese and control groups were matched with propensity scores by using all available baseline data. PSM was established through a multivariate logistic regression model, which considered the following variables: age, sex, trauma history, herniated segment, herniated type, preoperative visual analog scale (VAS) score, and Oswestry disability index (ODI) score. Matching was performed using the nearest-neighbor matching algorithm (caliper width 0.25 , standard deviation of the logit score) with a 1:1 ratio without replacement. Standardized mean differences (SMD) were compared before and after PSM to evaluate the matching of balanced potential confounders for the two study groups [9]. Covariates with a standardized difference of $<0.15$, in absolute value, were considered satisfactorily balanced.

\section{Surgical Procedure}

\section{PELD}


After the target disc was reconfirmed using C-arm X-ray fluoroscopy, the puncture point was marked. The puncture needle was sent to the target position, and a guidewire was inserted. A skin incision approximately $0.8 \mathrm{~cm}$ in length was made around the puncture site. Then, the dilating sleeve was placed along the guidewire step-by-step. Finally, we placed the intervertebral foraminoscope after reconfirming the correct position using C-arm X-ray fluoroscopy. Before we formally started the core step of surgery, lumbar discography was performed to identify the fibrous ring crack. The diseased nucleus pulposus tissue was removed using an endoscope, and the dural sac and nerve roots were thoroughly decompressed and loosened until spontaneous pulsation was achieved. Complete hemostasis was achieved with the assistance of bipolar radiofrequency, and the fibrous ring crack was repaired. The endoscope was moved along the nerve root, and the operative field was carefully examined to confirm that no residual compression was present. After endoscope removal, the incision was closed using sutures. The operation was completed without a wound drainage tube.

\section{Postoperative treatment}

In addition to the temporary use of antibiotics during surgery, the patients did not routinely use antibiotics after the operation. The patients were regularly administered symptomatic treatments for nutritional nerves, dehydration, and analgesia. Patients wearing waist circumference were allowed to leave the sickbed $6 \mathrm{~h}$ postoperatively. Patients were instructed to rest in bed for 1 month after surgery and to wear waist circumference during daily activities, and to avoid standing for long periods of time or performing physical labor within 3 months of surgery.

Patients were followed up in the outpatient clinic at 3 months, 6 months, and 1 year after surgery. Afterwards, it was recommended that patients be followed at least once per year. The re-examination items mainly included computed tomography or MRI of the lumbar spine.

\section{Clinical Evaluation and Follow-Up}

Demographic and perioperative clinical data were obtained by consulting surgical records and electronic medical records. In addition, we collected data on disease recurrence and complications through telephone and outpatient follow-up. The demographic data included sex, age, BMl, duration of symptoms, and follow-up time. Perioperative data included operative time, intraoperative blood loss and length of postoperative hospitalization. The major indicators for evaluating the efficacy of PELD included the VAS score [10] for lower back and leg pain, the ODI score [11], and modified MacNab criteria [12] obtained at the last follow-up timepoint. The minor indicators included the complication and recurrence rates obtained from the medical records or the last follow-up timepoint. The VAS score was collected before surgery, the first day after surgery, 3 months after surgery, and at the last follow-up timepoint. The evaluation of sexual function was excluded given the sensitive nature of this topic in the adolescent population, and the ODI questionnaire included nine items. In addition, considering that many items in the ODI questionnaire cannot be accurately reflected during the postoperative period in the hospital due to pain or medical advice, the ODI evaluation in this study were only performed before surgery, 3 months after surgery and at the last follow-up timepoint. The modified MacNab criteria and its associated clinical efficacy classification were used to evaluate the clinical efficacy of the two groups at the last follow-up timepoint. According to the theory proposed by Kraemer et al. [13], we classified the complications of lumbar spine surgery into intraoperative complications such as incorrect level, nerve root lesion, immediate postoperative complications (postoperative complications during hospitalization) such as residual symptom and new symptom, and late postoperative complications (complications after 
discharge). The judgment of recurrence was mainly based on the lumbar MRI diagnosis of the patient after the onset of symptoms or the second surgical treatment.

\section{Statistical Analysis}

All data analyses were performed using SPSS version 26.0 (IBM, Armonk, New York, USA). Categorical data are expressed as the number of cases or percentages, and the differences between the two groups were analyzed using the $\chi^{2}$ test or Fisher's exact probability method. Normally distributed measurement data are expressed as the mean \pm standard deviation, and Student's t-test was used to compare differences between the two groups. One-way analyses of variance were used for the comparison of VAS or ODI scores within the same group at different times, and the least significant difference method was used for pairwise comparison. Moreover, measurement data that were not normally distributed were expressed by the median and interquartile range, and analyzed using the Mann-Whitney $U$ test to observe difference between the two groups. Differences were considered to be statistically significant at $p<0.05$.

\section{Results}

A total of 208 subjects were included in the study, including 57 patients in the obese group and 151 patients in the control group, and 174 patients (83.7\%) were followed up. There were 9 patients (15.8\%) and 25 patients (16.6\%) lost to follow-up in the obese and nonobese groups, respectively. However, there was no significant difference in the lost to follow-up rate between the two groups $(p=0.894)$. After excluding patients who were lost to follow-up, there were 48 patients in the obese group and 126 patients in the control group. Finally, 45 patients were included in each group after 1:1 PSM. The follow-up time ranged from 12 to 70 months. The follow-up time ranged from 12 to 70 months, with a mean follow-up time of $28.44 \pm 16.41$ months in the obese group and $27.09 \pm 13.80$ months in the control group.

The basic preoperative characteristics of each group before and after PSM are shown in Table 1. There were significant differences in preoperative VAS scores between the two groups before PSM $(p<0.05)$. However, the balance of each variable was significantly improved after PSM ( $|S M D|<0.15$, Table 1), and the baseline data of the two groups were consistent $(p>0.05$, Table 1$)$. 
Table 1

Comparison of preoperative basic characteristics between the two groups before and after 1:1 PSM

\section{Before PSM}

Characteristic

$\begin{array}{ll}\text { Obese } & \text { Control } \\ (n=48) & (n=126)\end{array}$

Sex, $\mathrm{n}$

$(\%)$

\begin{tabular}{l} 
Male \\
\multicolumn{1}{c}{ Female } \\
Age (years), mean \pm \\
$\begin{array}{l}\text { Duration of } \\
\text { symptoms(months), } \\
\text { M (IQR) }\end{array}$
\end{tabular}

Trauma history, $\mathrm{n}$

$(\%)$

\begin{tabular}{lll} 
Yes & $9(18.8)$ & $35(27.8)$ \\
\hline No & $39(81.3)$ & $91(72.2)$
\end{tabular}

$0.221-0.229$

$0.796-0.056$

After 1:1 PSM

$\begin{array}{lllll}P & \text { SMD } & \text { Obese } & \text { Control } & P \\ \text { value } & & \text { value } & \text { SMD } \\ & & & \end{array}$

$0.156 \quad 0.269$

$1.000 \quad 0.000$

39(81.3) 89(70.6)

36(80.0) 36(80.0)

9(18.8) 37(29.4)

$9(20.0) \quad 9(20.0)$

$\begin{array}{ll}18.52 \pm & 18.76 \pm \\ 2.53 & 2.29\end{array}$

$0.548 \quad-0.095$

$18.44 \pm \quad 18.53 \pm$

2.55

2.41

$0.866-0.035$

$\begin{array}{llllllll}6(3.25- & 6(3-12) & 0.920 & -0.003 & 6(3-11) & 6(3-12) & 0.669 & 0.000\end{array}$

12)

$0.221-0.229$

(2)

No

Herniated segment,

n (\%)

\begin{tabular}{lll}
\hline L4-L5 & $28(58.3)$ & $61(48.4)$ \\
\hline L5-S1 & $19(39.6)$ & $61(48.4)$ \\
$\begin{array}{l}\text { Other } \\
\text { level }\end{array}$ & $1(2.1)$ & $4(3.2)$ \\
\hline
\end{tabular}

Herniated type, $\mathrm{n}$

$(\%)$

$0.549-0.203$

$\begin{array}{ll}9(20.0) & 10(22.2) \\ 36(80.0) & 35(77.8)\end{array}$

$1.000 \quad 0.000$

\begin{tabular}{|c|c|c|c|c|c|c|c|c|}
\hline Lateral & $22(45.8)$ & $72(57.1)$ & & & $22(48.9)$ & $19(42.2)$ & & \\
\hline Central & $26(54.2)$ & $54(42.9)$ & & & $23(51.1)$ & $26(57.8)$ & & \\
\hline $\begin{array}{l}\text { Preoperative VAS, } \\
\text { mean } \pm \text { SD }\end{array}$ & $\begin{array}{l}6.35 \pm \\
1.19\end{array}$ & $\begin{array}{l}6.73 \pm \\
1.09\end{array}$ & 0.049 * & -0.315 & $\begin{array}{l}6.42 \pm \\
1.20\end{array}$ & $\begin{array}{l}6.51 \pm \\
0.94\end{array}$ & 0.697 & -0.074 \\
\hline $\begin{array}{l}\text { Preoperative ODI, } \\
\text { mean } \pm \text { SD }\end{array}$ & $\begin{array}{l}59.25 \pm \\
62.12\end{array}$ & $\begin{array}{l}62.12 \pm \\
9.35\end{array}$ & 0.074 & -0.300 & $\begin{array}{l}59.38 \pm \\
9.81\end{array}$ & $\begin{array}{l}58.802 \\
\pm 9.47\end{array}$ & 0.777 & 0.060 \\
\hline
\end{tabular}

PSM, propensity score matching; SMD, standardized mean difference; BMI, body mass index; VAS, visual analog scale; ODI, Oswestry disability index;

SD, standard deviation; IQR, Interquartile range; M, Median

*significant difference between two groups 
A comparison of preoperative data between the two groups is shown in Table 2. There was no significant difference between the two groups in terms of operative time, intraoperative blood loss, and length of postoperative hospitalization $(p>0.05)$.

Table 2

Comparison of perioperative data between the two groups

\begin{tabular}{|llll|}
\hline Parameter & $\begin{array}{l}\text { Obese } \\
(\mathbf{n = 4 5 )}\end{array}$ & $\begin{array}{l}\text { Control } \\
(\mathbf{n = 4 5 )}\end{array}$ & Pvalue \\
\hline Surgery duration(minutes), M (IQR) & $46(32 \sim 66.5)$ & $50(37.5 \sim 73.5)$ & 0.159 \\
\hline Intraoperative Blood loss (ml), M (IQR) & $5(2.5 \sim 5)$ & $5(5 \sim 5)$ & 0.278 \\
\hline Duration of postoperative hospitalization(days), M (IQR) & $3(2 \sim 3)$ & $3(2 \sim 3)$ & 0.954 \\
\hline IQR, Interquartile range; M, Median & & & \\
\hline
\end{tabular}

The comparison of clinical outcome indicators between the two groups is shown in Table 3. The two groups had no significant differences in the main indicators of clinical outcomes, such as VAS scores, ODI scores at each postoperative time point and the distribution of the MacNab criteria assessments $(p>0.05)$. In accordance with the modified MacNab standard classification, the excellence and good rate of the obese and control groups were $93.3 \%$ and $97.8 \%$, respectively, which were also not statistically significant $(p>0.05)$. 
Table 3

Comparison of the indicators of clinical outcome between the two groups

\begin{tabular}{|c|c|c|c|}
\hline Parameter & $\begin{array}{l}\text { Obese } \\
(n=45)\end{array}$ & $\begin{array}{l}\text { Control } \\
(n=45)\end{array}$ & $P$ value \\
\hline \multicolumn{4}{|c|}{ VAS for lower back and leg pain, mean \pm SD } \\
\hline Preoperative & $6.42 \pm 1.20$ & $6.51 \pm 0.94$ & 0.697 \\
\hline 1 day postoperative & $1.82 \pm 1.07$ & $1.89 \pm 0.88$ & 0.748 \\
\hline 3 months postoperative & $0.78 \pm 1.17$ & $0.60 \pm 0.84$ & 0.408 \\
\hline Final follow-up & $0.49 \pm 0.99$ & $0.27 \pm 0.62$ & 0.205 \\
\hline \multicolumn{4}{|l|}{ ODI(\%), mean \pm SD } \\
\hline Preoperative & $59.38 \pm 9.81$ & $58.802 \pm 9.47$ & 0.777 \\
\hline 3 months postoperative & $7.98 \pm 4.05$ & $6.93 \pm 1.96$ & 0.123 \\
\hline Final follow-up & $7.93 \pm 4.30$ & $6.98 \pm 1.91$ & 0.177 \\
\hline Modified MacNab, n (\%) & & & 0.631 \\
\hline Excellence & $32(71.1)$ & $35(77.8)$ & \\
\hline Good & $10(22.2)$ & $9(20.0)$ & \\
\hline Fair & $3(6.7)$ & $1(2.2)$ & \\
\hline Poor & $0(0)$ & $0(0)$ & \\
\hline Clinical efficacy classification, n (\%) & & & 0.609 \\
\hline Excellence and good & 42(93.3) & $44(97.8)$ & \\
\hline Fair and poor & $3(6.7)$ & $1(2.2)$ & \\
\hline Complications, n (\%) & & & 0.789 \\
\hline Intraoperative & 0 & 0 & \\
\hline Immediate postoperative & $1(2.2)$ & $0(0)$ & \\
\hline Late postoperative & $8(17.8)$ & $7(14.8)$ & \\
\hline
\end{tabular}

In terms of the VAS score changes during the follow-up period (Fig. 2, Table 3), the VAS scores on the first postoperative day, 3 months postoperatively, and at the final follow-up timepoint were significantly lower than the preoperative VAS scores in both the obese and control groups $(p<0.05)$. Regarding the ODI score changes during the follow-up period (Fig. 3, Table 3 ), the ODI scores at 3 months postoperatively and at the final follow- 
up timepoint were significantly lower than the preoperative VAS scores in both groups $(p<0.05)$. There was no significant difference in the postoperative VAS score and ODI score between the two groups (Fig. 2 and Fig. 3).

Based on the minor indicators of clinical outcomes of the two groups, there was no significant difference in the overall incidence rate of complications between the two groups $(20 \%$ vs. $15.6 \%, p=0.581)$. There were no intraoperative complications in either group. However, one patient had immediate postoperative complications. The patient felt that the symptoms of preoperative lower back pain were basically relieved after returning to the ward, but the pain of the right lower limb was not relieved completely, with the symptom of hyperesthesia on the lateral side of right lower leg but no neurological symptoms or cauda equina syndrome. Finally, the above symptoms gradually relieved one year later after he took painkillers and neurotrophic drugs intermittently. However, there were no immediate postoperative complications in the control group. Moreover, eight patients had late postoperative complications in the obese group. Seven of these patients complained that they felt intermittent pain or discomfort in their lower back or buttock (VAS scores of 2-3), especially when they were tired, and most of their pain could be relieved itself without oral analgesics after were sufficiently rested. Only one patient developed more severe pain with no neurological symptoms (VAS scores of 5-6) 1 year after surgery, and this patient went to the local hospital and excluded the possibility of recurrence. Notably, this patient experienced no obvious pain after treatment with Chinese medicine. The conditions in the control group were similar to those in the obese group. Six patients in the control group experienced intermittent postoperative mild pain symptoms without oral analgesics, but only one patient required oral analgesics intermittently. Furthermore, no recurrence was observed in either group at the last follow-up timepoint.

\section{Discussion}

According to the characteristics of the spinal growth and development process, the vertebral epiphyseal cartilage is normally completely fused with the vertebral body around 21 years of age [14]; hence, 21 years of age was defined as the upper age limit for patients with ALDH in this study. However, the lower age limit has not been clearly defined. Raghu et al. [15] reported that LDH patients under 12 years of age were very rare, and the youngest patient in this study was a 10-year-old girl. BMI is one of the commonly used standards for measuring human body fat. $\mathrm{A} \mathrm{BMI} \geq 28 \mathrm{~kg} / \mathrm{cm}^{2}$ is used as the diagnostic criterion for obese patients over 18 years of age in our country. However, since adolescents under 18 years of age are still growing and developing, the above criteria for obesity are not suitable for the adolescent population. Therefore, we classified obese adolescent patients according to the obesity classification criteria for adolescents and children under 18 years of age in this study.

Over the past 30 years, obesity has become a global epidemic that threatens public health. The prevalence of obesity and overweight increased by $27.5 \%$ in adults and $47.1 \%$ in children from 1980 to 2013, and the number of overweight and obese people increased from 921 million in 1980 to 2.1 billion in 2013[16]. The prevalence of obesity in children and adolescents had increased significantly. Numerous studies have suggested that obesity is associated with the incidence of various diseases such as cardiovascular disease, cancer, and bone and joint diseases [17-19], and there is evidence that obesity is also an important risk factors for the onset of ALDH [4,20]. Obesity applies an excessive load to the intervertebral disc, and it leads to an abnormal inflammatory response and endocrine regulation in the human body, which eventually resulted 
in accelerated degeneration or damage to intervertebral disc. Obese adolescents accounted for $27.4 \%$ of patients with ALDH patients in our study, so we should closely observe obese people with such diseases whether they are adults or adolescents.

spinal surgery in obesity is a challenging endeavor for many reasons, including anesthesia, intravenous access, positioning, and wound exposure. Most spine surgeons would agree that surgical intervention is difficult in this population. Before the emergence of minimally invasive spinal surgery, this group of patients usually required longer surgical incisions than the general population to fully expose the surgical area during lumbar disc herniation, but many complications may occur, including wound infection or poor healing, which affected the surgical satisfaction of these individuals and surgical efficacy [21, 22]. With the development of minimally invasive techniques and the requirement of medical apparatus, PELD has emerged and is widely used in adult and adolescent spinal surgery. Numerous clinical studies have suggested that PELD not only has comparable efficacy compared with conventional spinal surgery, but also has the advantages of reduced blood loss, reduced tissue destruction, and faster postoperative recovery [23-25]. Moreover, several studies $[26,27]$ have suggested that the application of PELD in obese adults with LDH could reduce the incidence of complications such as wound infection or poor healing. We assume that the decreased incidence of complication in obese patients with PELD can be attributed to technological breakthroughs in the deep surgical field; operations require less time and thus, decrease the chances of contamination and paraspinal muscles trauma. In addition, PELD is usually performed under local anesthesia; therefore, general anesthesiarelated adverse events can be avoided effectively. However, no studies have evaluated the efficacy of PELD in obese patients with ALDH.

Compared with adult patients with LDH, patients with ALDH also have the following characteristics. First, the surgical methods is cautiously selected. Regardless of the surgical method used, we need to focus on minimizing the surgical impact on spinal growth and development and the possibility of secondary adjacent segment degeneration or recurrent disc herniation after surgery [28]. Compared with open surgery, PELD minimizes structural damage in the normal spine, such as muscles and facet joints, and reduces the recurrence rate of postoperative iatrogenic instability. Second, the scope of surgical resection is limited. Whether it is PELD or open surgery, the scope of discectomy in adolescents should be controlled to create conditions for the regeneration of intervertebral discs [29] and maximize the retention of the remaining disc function. Finally, it is necessary to consider whether the growth and development of the adolescent spine affects the efficacy of surgery. Gulati et al. [30] believed that the growth of adolescent spine may affect the efficacy of surgery. However, our study showed that in obese or non-obese patients, the excellent and good rate of surgery was more than $93 \%$. Given that PELD is more complicated than traditional open spinal surgery and there may be complications such as residual nucleus pulposus, intraspinal hematoma, infection, and more [31], it often requires a longer learning curve to maximize its performance[32].

Based on the basic characteristics of the two groups before surgery, we found that there were no significant differences in age, sex, history of trauma, segment and type of herniation, severity of preoperative lower back and leg pain, and ODI scores after PSM, so the comparison between the two groups was more reliable. In terms of perioperative data such as operative time, intraoperative blood loss and length of postoperative hospitalization, there was no statistical difference between the obese and control groups (Table $3, P>0.05$ ), indicating that compared with non-obese patients, obese patients with ALDH may accept PELD without 
significant difficulties. We believe that because PELD caused less surgical trauma and required no wound drainage tubes, it can effectively shorten the postoperative bedridden recovery time, reduce the occurrence of bed-related complications and hospitalization costs, improve postoperative quality of life, and help patients return to their normal life or work faster, which is in line with the current concept of enhanced recovery after surgery in the field of spinal surgery [33]. However, this was contrary to the results reported in some studies that microdiscectomy or open surgery increased the amount of intraoperative blood loss and length of hospitalization in obese adults $[7,34]$, indicating that PELD was advantageous in the treatment of obese patients with $\mathrm{LDH}$ from the other side.

Based on the main indicators of clinical outcome, the postoperative VAS and ODI scores of both groups were significantly lower than the preoperative scores (Fig. 2, Fig. 3, p<0.05), and there was no difference in VAS and ODI scores between the two groups at each follow-up time (Table $3, p>0.05$ ). This indicated that the clinical efficacy of PELD in both obese and non-obese adolescents was comparable, and not affected by obesity. Rihn et al. [6] conducted a 4-year follow-up study of 854 nonobese and 336 obese adults and found that obese patients had less improvement in pain symptoms or postoperative ODI scores than non-obese patients. But the researcher did not clearly explain the surgical method used. Although their results in adults differed from our results in adolescents, it may also suggest that the efficacy of PELD in adolescent patients may not be affected by obesity. From the perspective of minor indicators of clinical outcome, there were no serious complications in either group, and the overall incidence of complications and recurrence rate were not significantly different between the two groups (Table $3, p>0.05$ ). However, Meredith et al. [35] believed that obesity was a strong and independent predictor of recurrence in patients who underwent lumbar discectomy, which was also inconsistent with our results. We believe that the following two aspects may explain this difference. First, the follow-up time in our study was not sufficiently long, and there was a certain proportion of patients who were lost to follow-up. Second, most adolescents were highly compliant and may adopt more strict postoperative rehabilitation plans under the supervision of their parents. Moreover, the postoperative complications of PELD in our study were mainly manifested as recurring lower back pain or insignificant relief of postoperative pain symptoms, which existed in both the obesity and control groups. However, pain in most patients had little effect on their daily lives or required analgesic intervention. We believe that this finding may be related to abnormal expression of pro-inflammatory cytokines in the blood of patients after surgery or adjacent joint diseases [36, 37].

However, here are some limitations to our study. First, a retrospective single-center study design was used. The number of patients included and the follow-up time were limited, and there were some patients that we could not obtain follow-up data on, which lessened the accuracy of the present cohort. Therefore, a prospective randomized controlled trial with a larger sample size is needed to confirm our results.

\section{Conclusion}

In summary, this retrospective study suggested that PELD is a safe and effective minimally invasive technique for the treatment of obese patients with ALDH. The improvement of pain and disability in obese patients with ALDH were comparable with that in non-obese patients with ALDH, and there was no difference in intraoperative blood loss, operative time, and length of postoperative hospitalization between these groups. Obese patients with ALDH who undergo PELD do not face a greater risk of complications and recurrence, 
indicating that PELD has good short- and mid-term effects. However, a prospective randomized controlled trial with a larger sample size is still needed to further confirm our results.

\section{Abbreviations}

$\mathrm{BMI}=$ body mass index; PELD = percutaneous endoscopic lumbar discectomy; $\mathrm{LDH}$ = lumbar disc herniation; ALDH = adolescent lumbar disc herniation; VAS = visual analog scale; ODI囚oswestry disability index; SMD standardized mean difference; PSM \propensity score matching; MRI『magnetic resonance imaging; SD区 standard deviation; IQR囚interquartile range; M『median; RCT『randomized controlled trial

\section{Declarations}

\section{Competing interests}

The authors declare they have no competing or conflict of interest.

\section{Ethics approval and consent to participate}

The study was conducted in accordance with the Declaration of Helsinki and was approved by the Peking University Third Hospital Medical Science Research Ethics Committee. Because of the retrospective nature of the study, patient consent for inclusion was waived.

\section{Consent for publication}

Not applicable.

\section{Funding}

The study was not funded

\section{Acknowledgements}

We would like to thank Editage (www.editage.cn) for English language editing.

\section{Availability of data and materials}

The datasets used and/or analysed during the current study are available from the corresponding author on reasonable request.

\section{Authors' contributions}

All authors contributed to the study conception and design. Material preparation, data collection and analysis were performed by Haijiang Yu. The data was evaluated by Bin Zhu and Xiaoguang Liu. The first draft of the manuscript was written by Haijiang Yu and Bin Zhu. All authors read and approved the final manuscript.

\section{Author details}


${ }^{1}$ Department of Orthopedics, Peking University Third Hospital, Beijing, China

${ }^{2}$ Department of Orthopedics, Capital Medical University Affiliated Beijing Friendship Hospital, Beijing, China

\section{References}

1. Lee JY, Ernestus RI, Schröder R, Klug N. Histological Study of Lumbar Intervertebral Disc Herniation in Adolescents. Acta Neurochir (Wien). 2000;142(10):1107-10. https://doi.org/10.1007/s007010070037

2. Ozgen S, Konya D, Toktas OZ, Dagcinar A, Ozek MM. Lumbar disc herniation in adolescence. Pediatr Neurosurg 2007;43(2):77-81. https://doi.org/10.1159/000098377

3. Wang H, Cheng J, Xiao H, Li C, Zhou Y. Adolescent lumbar disc herniation: Experience from a large minimally invasive treatment centre for lumbar degenerative disease in Chongqing, China. Clin Neurol Neurosurg. 2013;115(8):1415-9. https://doi.org/10.1016/j.clineuro.2013.01.019

4. Mattila VM, Saarni L, Parkkari J, Koivusilta L, Rimpelä A. Early risk factors for lumbar discectomy: an 11year follow-up of 57,408 adolescents. Eur Spine J. 2008;17(10): 1317-23. https://doi.org/10.1007/s00586-008-0738-2

5. Abdallah DY, Jadaan MM, McCabe JP. Body mass index and risk of surgical site infection following spine surgery: a meta-analysis. Eur Spine J. 2013;22(12):2800-9. https://doi.org/10.1007/s00586-013-2890-6

6. Rihn JA, Kurd M, Hilibrand AS, Lurie J, Zhao W, Albert T, et al. The influence of obesity on the outcome of treatment of lumbar disc herniation: analysis of the Spine Patient Outcomes Research Trial (SPORT). J Bone Joint Surg Am. 2013;95(1):1-8. https://doi.org/10.2106/JBJS.K.01558

7. Jiang J, Teng Y, Fan Z, Khan S, Xia Y. Does obesity affect the surgical outcome and complication rates of spinal surgery? A meta-analysis. Clin Orthop Relat Res. 2014;472(3):968-75. https://doi.org/10.1007/s11999-013-3346-3

8. Cole JS 4th, Jackson TR. Minimally invasive lumbar discectomy in obese patients. Neurosurgery. 2007;61(3):539-44. https://doi.org/10.1227/01.NEU.0000290900.23190.C9

9. Austin PC. Balance diagnostics for comparing the distribution of baseline covariates between treatment groups in propensity-score matched samples. Stat Med. 2009;28(25):3083-

10https://doi.org/10.1002/sim.3697

10. Huskisson EC. Measurement of pain. Lancet 1974;2(7889):1127-31. https://doi.org/10.1016/s01406736(74)90884-8

11. Fairbank JC, Couper J, Davies JB, O'Brien JP. The Oswestry low back pain disability questionnaire. Physiotherapy. 1980;66(8):271-3.

12. Macnab I. Negative disc exploration. An analysis of the causes of nerve-root involvement in sixty-eight patients. J Bone Joint Surg Am. 1971;53(5):891-903.

13. Kraemer R, Wild A, Haak H, Herdmann J, Krauspe R, Kraemer J. Classification and management of early complications in open lumbar microdiscectomy. Eur Spine J. 2003;12(3):239-46. https://doi.org/10.1007/s00586-002-0466-y

14. Silvers HR, Lewis PJ, Clabeaux DE, Asch HL. Lumbar disc excisions in patients under the age of 21 years. Spine (Phila Pa 1976). 1994;19(21):2387-91. https://doi.org/10.1097/00007632-199411000-00002 
15. Raghu ALB, Wiggins A, Kandasamy J. Surgical management of lumbar disc herniation in children and adolescents. Clin Neurol Neurosurg. 2019;185:105486. https://doi.org/10.1016/j.clineuro.2019.105486

16. Ng M, Fleming T, Robinson M, Thomson B, Graetz N, Margono C, Mullany EC, et al. Global, regional, and national prevalence of overweight and obesity in children and adults during 1980-2013: a systematic analysis for the Global Burden of Disease Study 2013.Lancet. 2014;384(9945):766-81. https://doi.org/10.1016/S0140-6736(14)60460-8

17. Ni Mhurchu C, Rodgers A, Pan WH, Gu DF, Woodward M. Body mass index and cardiovascular disease in the Asia-Pacific Region: an overview of 33 cohorts involving 310000 participants. Int J Epidemiol. 2004 Aug;33(4):751-8. https://doi.org/10.1093/ije/dyh163

18. Renehan AG, Tyson M, Egger M, Heller RF, Zwahlen M. Body-mass index and incidence of cancer: a systematic review and meta-analysis of prospective observational studies. Lancet. 2008;371(9612):56978. https://doi.org/10.1016/S0140-6736(08)60269-X

19. Wormser D, Kaptoge S, Di Angelantonio E, Wood AM, Pennells L, Thompson A, et al. Separate and combined associations of body-mass index and abdominal adiposity with cardiovascular disease: collaborative analysis of 58 prospective studies. Lancet. 2011;377(9771):1085-95. https://doi.org/10.1016/S0140-6736(11)60105-0

20. Li Y, Shi JJ, Ren J, Guan HS, Gao YP, Zhao F, Sun J. [Relationship between obesity and lumbar disc herniation in adolescents]. Zhongguo Gu Shang. 2020;33(8):725-9. Chinese. https://doi.org/10.12200/j.issn.1003-0034.2020.08.008

21. De la Garza-Ramos R, Bydon M, Abt NB, Sciubba DM, Wolinsky JP, Bydon A, et al. The impact of obesity on short- and long-term outcomes after lumbar fusion. Spine(Phila Pa 1976).2015;40(1):56-61. https://doi.org/10.1097/BRS.0000000000000655

22. McGuire KJ, Khaleel MA, Rihn JA, Lurie JD, Zhao W, Weinstein JN. The effect of highobesity on outcomes of treatment for lumbar spinal conditions: subgroup analysis of the spine patient outcomes research trial. Spine (Phila Pa 1976). 2014;39(23):1975-80. https://doi.org/10.1097/BRS.0000000000000577

23. Li Z, Zhang C, Chen W, Li S, Yu B, Zhao H, et al. Percutaneous Endoscopic Transforaminal Discectomy versus Conventional Open Lumbar Discectomy for Upper Lumbar Disc Herniation: A Comparative Cohort Study. Biomed Res Int. 2020;2020:1852070. https://doi.org/10.1155/2020/1852070

24. Kim M, Lee S, Kim HS, Park S, Shim SY, Lim DJ. A Comparison of Percutaneous Endoscopic Lumbar Discectomy and Open Lumbar Microdiscectomy for Lumbar Disc Herniation in the Korean: A MetaAnalysis. Biomed Res Int. 2018;2018:9073460. https://doi.org/10.1155/2018/9073460

25. Li H, Jiang C, Mu X, Lan W, Zhou Y, Li C. Comparison of MED and PELD in the Treatment of Adolescent Lumbar Disc Herniation: A 5-Year Retrospective Follow-Up. World Neurosurg. 2018;112:e255-e260. https://doi.org/10.1016/j.wneu.2018.01.030

26. Bae JS, Lee SH. Transforaminal full-endoscopic lumbar discectomy in obese patients. Int J Spine Surg. 2016;10:18. https://doi.org/10.14444/3018

27. Wang YP, Zhang W, An JL, Zhang J, Bai JY, Sun YP. Evaluation of Transforaminal Endoscopic Discectomy in Treatment of Obese Patients with Lumbar Disc Herniation. Med Sci Monit. 2016;22:2513-9. https://doi.org/10.12659/msm.899510 
28. Haddadi K: Pediatric Lumbar Disc Herniation: A Review of Manifestations, Diagnosis and Management. Journal of Pediatrics Review. 2016;4(1):0-0. https://doi.org/10.17795/jpr-4725

29. Bayliss MT, Johnstone B, O'Brien JP. 1988 Volvo award in basic science. Proteoglycan synthesis in the human intervertebral disc. Variation with age, region and pathology. Spine (Phila Pa 1976). 1988;13(9):972-81. https://doi.org/10.1097/00007632-198809000-00003

30. Gulati S, Madsbu MA, Solberg TK, Sørlie A, Giannadakis C, Skram MK, et al. Lumbar microdiscectomy for sciatica in adolescents: a multicentre observational registry-based study. Acta Neurochir (Wien). 2017;159(3):509-516. https://doi.org/10.1007/s00701-017-3077-4

31. Xu Z, Liu Y, Chen J. Percutaneous Endoscopic Interlaminar Discectomy for L5-S1 Adolescent Lumbar Disc Herniation. Turk Neurosurg 2018;28(6):923-928. https://doi.org/10.5137/1019-5149.JTN.21579-17.3

32. Ahn SS, Kim SH, Kim DW. Learning Curve of Percutaneous Endoscopic Lumbar Discectomy Based on the Period (Early vs. Late) and Technique (in-and-out vs. in-and-out-and-in): A Retrospective Comparative Study. Journal of Korean Neurosurgical Society 2015;58(6):539-546.

https://doi.org/10.3340/jkns.2015.58.6.539

33. D'Astorg H, Fière V, Dupasquier M, Vieira TD, Szadkowski M: Enhanced recovery after surgery (ERAS) protocol reduces LOS without additional adverse events in spine surgery. Orthop Traumatol Surg Res. 2020;106(6): 1167-1173. https://doi.org/10.1016/j.otsr.2020.01.017

34. Madsbu MA, Øie LR, Salvesen $\emptyset$, Vangen-Lønne V, Nygaard ØP, Solberg TK, et al. Lumbar Microdiscectomy in Obese Patients: A Multicenter Observational Study. World Neurosurg. 2018;110:e1004-e1010. https://doi.org/10.1016/j.wneu.2017.11.156

35. Meredith DS, Huang RC, Nguyen J, Lyman S. Obesity increases the risk of recurrent herniated nucleus pulposus after lumbar microdiscectomy. Spine J. 2010;10(7):575-80. https://doi.org/10.1016/j.spinee.2010.02.021

36. Inoue S, Kamiya M, Nishihara M, Arai YP, Ikemoto T, Ushida T. Prevalence, characteristics, and burden of failed back surgery syndrome: the influence of various residual symptoms on patient satisfaction and quality of life as assessed by a nationwide Internet survey in Japan. J Pain Res. 2017;10:811-823. https://doi.org/10.2147/JPR.S129295

37. Kamieniak P, Bielewicz J, Grochowski C, Litak J, Bojarska-Junak A, Daniluk B, et al. The Elevated Serum Level of IFN-y in Patients with Failed Back Surgery Syndrome Remains Unchanged after Spinal Cord Stimulation. Dis Markers. 2019;2019:2606808. https://doi.org/10. 1155/2019/2606808

\section{Figures}




\begin{tabular}{|c|c|c|}
\hline \multirow{2}{*}{$\begin{array}{c}\text { Age } \\
\text { (years) }\end{array}$} & \multicolumn{2}{|c|}{$\mathrm{BMI}\left(\mathrm{Kg} / \mathrm{cm}^{2}\right)$} \\
\hline & Male & Female \\
\hline 6 & 17.7 & 17.5 \\
\hline $7 \sim$ & 18.7 & 18.5 \\
\hline 8 & 19.7 & 19.4 \\
\hline 9 & 20.8 & 20.4 \\
\hline $10 \sim$ & 21.9 & 21.5 \\
\hline $11 \sim$ & 23.0 & 22.7 \\
\hline $12 \sim$ & 24.1 & 23.9 \\
\hline 13 & 25.2 & 25.0 \\
\hline 14 & 26.1 & 25.9 \\
\hline $15 \sim$ & 26.6 & 26.6 \\
\hline $16 \sim$ & 27.1 & 27.1 \\
\hline $17 \sim$ & 27.6 & 27.6 \\
\hline 18 & 28.0 & 28.0 \\
\hline
\end{tabular}

\section{Figure 1}

BMI threshold screening for obesity in children and adolescents aged $6 \sim 18$ years BMI, body mass index 


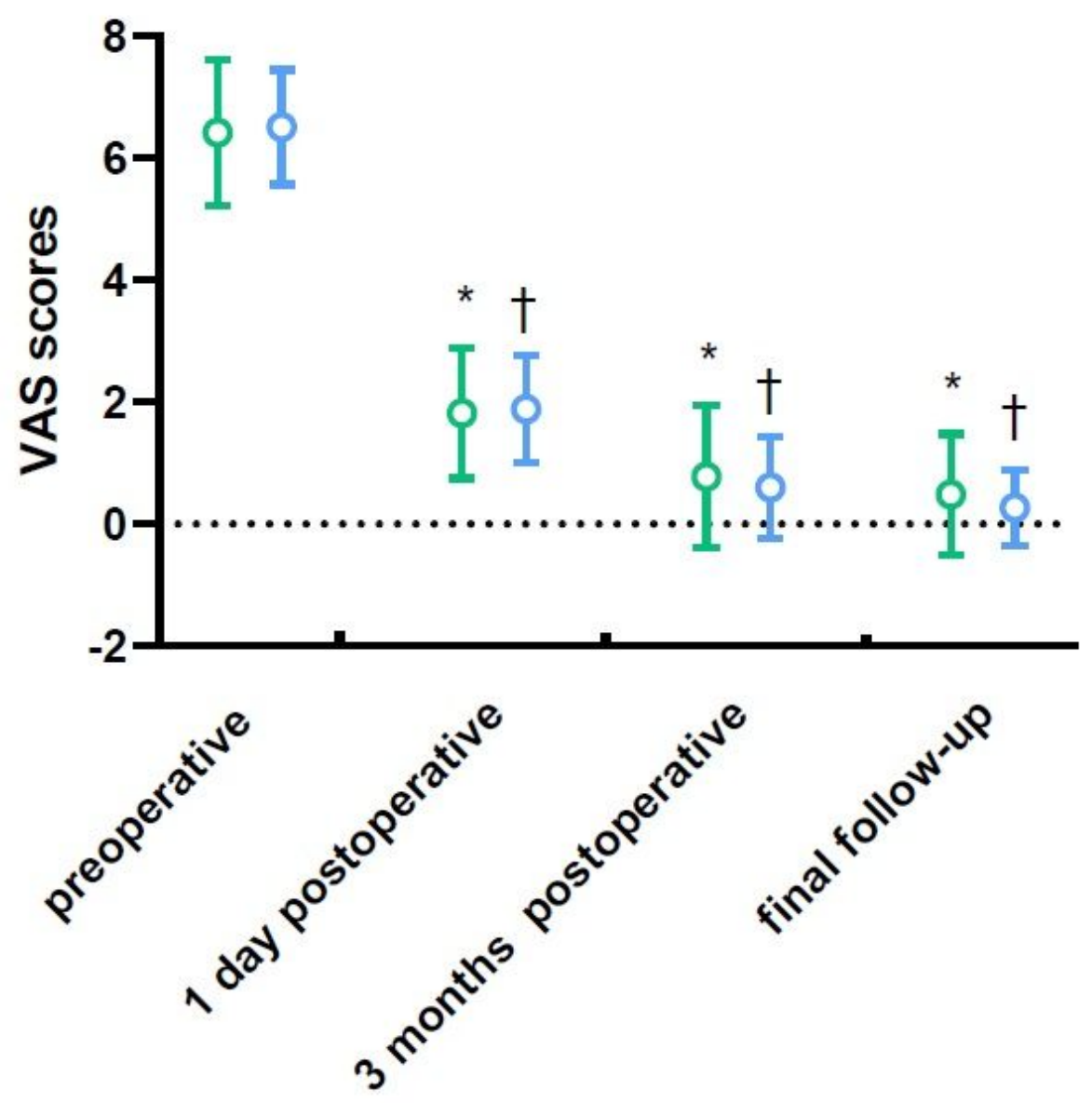

\section{1어 Obese (mean $\pm S D)$ ⺊어 Control(mean $\pm S D)$}

\section{Figure 2}

The change of VAS for lower back and leg pain during follow-up. VAS, visual analog scale; SD, standard deviation *Significant difference between preoperative and postoperative time in the obese group +Significant difference between the preoperative period and period at each follow-up time in the control group 


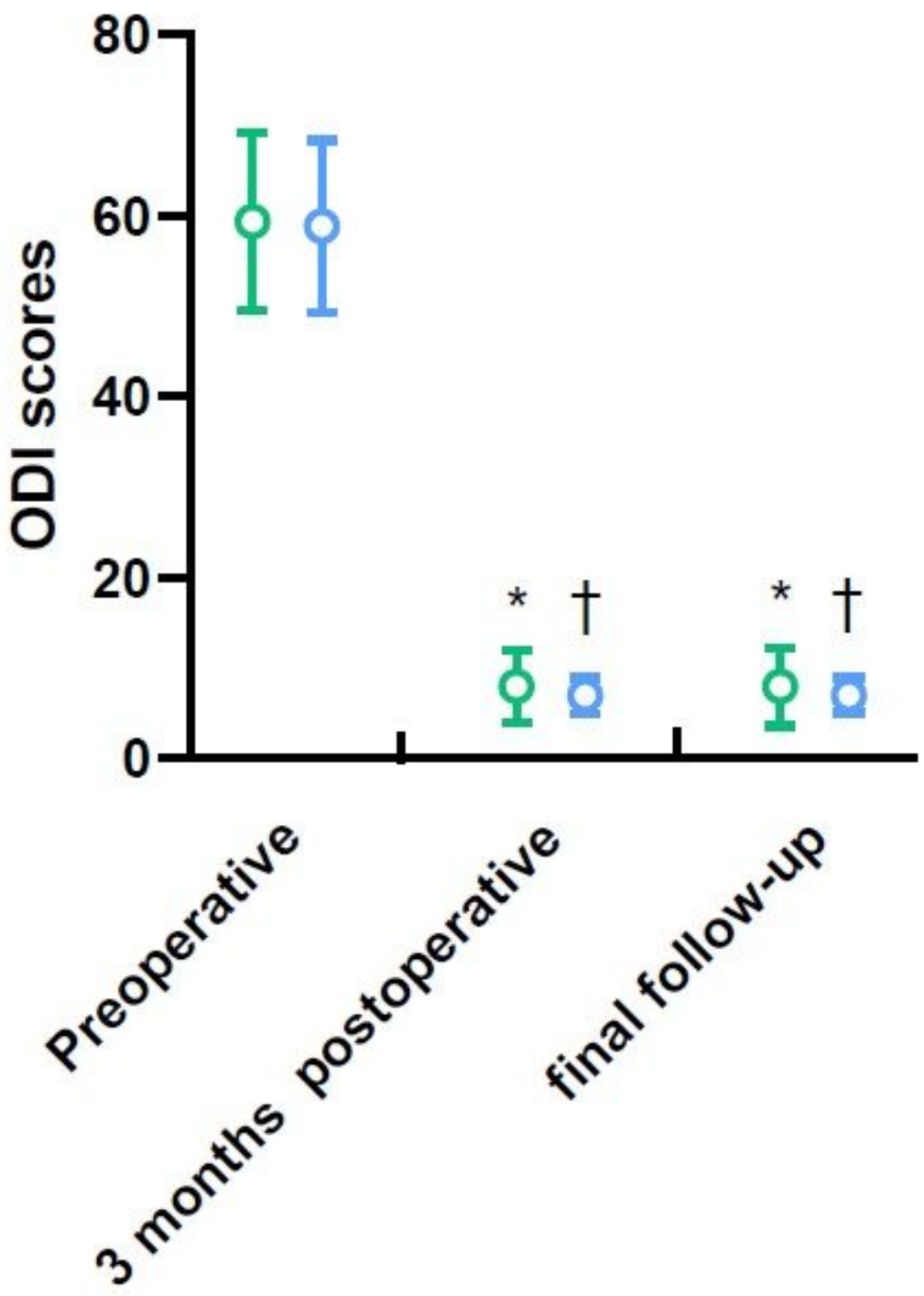

r어 Obese(mean $\pm S D)$

ro- Control(mean $\pm S D)$

Figure 3

The change of ODI during follow-up. ODI, oswestry disability index; SD, standard deviation *Significant difference between preoperative and postoperative time in the obese group +Significant difference between the preoperative period and period at each follow-up time in the control group 